\title{
Investigations on Restoration Scheme and Alarm Analysis in Power System
}

\author{
Arvind Singh Rathore \\ Department of Electrical Engineering \\ Sir Padampat Singhania University \\ Udaipur(Raj.),India
}

\author{
Prasun Chakrabarti \\ Department of Computer Engineering \\ Sir Padampat Singhania University \\ Udaipur(Raj.),India
}

\begin{abstract}
-
The work introduces Island Restoration Scheme (IRS) with online restoration of missing sensor data. This paper focuses on the Restoration Scheme with Intelligent Sensing and Monitoring and also entails fuzzy logic theory representation for alarm analysis in electrical power system based on expert system . Theoretical and mathematical representation of alarm analysis for fault diagnosis has also been pointed out..
\end{abstract}

\section{Keywords}

Restoration Scheme; Alarm Analysis; Auto Encoder; Artificial Neural Networks.

\section{INTRODUCTION}

The Restoration Scheme is made of two artificial neural networks. Each artificial neural network play important role in island restoration scheme. The number of IRSs will be defined by offline studies and will depend on the regional load-generation balance. This scheme is commonly used in transmission system where parallel restoration is more efficient and desired. The parallel restoration techniques are commonly used in the restoration schemes applied to large transmission systems. These techniques also used in the proposed restoration schemes. For switching operation strategy where all circuit breakers of the system are open will be used to create the islands. In order to restore a power system following a wide-area disturbance and IRS will play important role in the restoration scheme, it will generate local restoration plans composed of switching sequences of local circuit breakers and a forecast restoration load. So this is the basic idea that how is the IRS play the important role in the island restoration scheme. Now this paper explains about how to apply smart system concept in this scheme. So for this we are used the concept of automated asset monitoring. The smart system for alarm analysis for power system based on expert system is explained by mathematical concept. The restoration scheme is totally depend upon the some parameters like monitoring and control of any devices or sub systems in a smart grid and these parameters are depend on the quality and availability of sensor measurements. Due to unexpected system conditions of sensors, may result in incorrect operations of device and it is also affect the operation and reliability of power system. As we know that important aspect of smart grids, development of sensors and communications brings the opportunity of increasing the visibility of traditional power grids so we use a large amount of power system measurement data is not an easy task and that's why we use CI techniques have been applied to assist in this area. In the power system the fault generally happen, in power system two types of faults generally happen. First is small disturbances fault and second one is transient fault. So these Fault affect the power system operation. They activates the relay protection system which isolates the faulted zone. The triggering of certain protection sends a signal to a local transformer substation and a long-range signal to the dispatch centre. Alarms are visible through the scada system which alongside a remote control, measurements and monitoring alongside remote control. Alarm consist of a wide group of signals which comprise, signalling the position of the circuit breaker and the disconnected, their assembling condition is described with, the activation and the tripping of certain protection. It include failure of certain automatic switches inside the secondary development, failure of some devices in the same system, up to some minor alarms such as the signal high shows when transformer substation doors are bring opened. At the moment of the fault, the alarm play important role. It appear on the screen and they are positioned above each other, so the alarm on the top points out the last situations is manageable. If some critical situations occur like transient fault, it causes an avalanche of a few hundreds alarms within a short period of time, further complicating the situation, and thus disabling the operators' reaction in due time or leading him towards doing a wrong action and consequently towards immense damage. The transient fault which cannot be eliminated by the protection due to additional equipment damage, will thus trigger the secondary protection to do the job, leaving black out areas suspicion. During the fault, the operator has to process a huge amount of unprocessed data and carry out a complex decision making process within a short period of the time. In this type of situation we are used the alarm processors. The alarm processor uses the expert system as its computer programme. It is a computer programme which substitutes an experts thinking process within a narrow action field, to solve complex situations. As we know that the expert system consists of the knowledge base, algorithms and communication interface. The occurrence of fault is an unwanted situation like small disturbances and transient disturbances are in electric power systems where certain network parts detach themselves from the remaining unaffected part of the network. The relay protection system has a task to isolate the faulty units or zone. These types of situation we are totally depend on the dispatch centre. When a certain protection level is being triggered alarms are sent to the transformer station and the dispatcher centre. These alarm reach the dispatcher centre in a short period of time which can lead the dispatcher to confusion and possible mistakes.

\section{ISLAND RESTORATION SCHEME WITH ONLINE RESTORATION OF MISSING SENSOR DATA}

Each IRS is composed of two Artificial Neural Networks(ANN). The first ANN of each IRS is responsible for an island restoration load forecast[1]. The input of this ANN will be a normalized vector composed of the pre disturbance load. The second ANN of each IRS is responsible for the determination of the final island configuration and the associated forecast restoration load provided by the first ANN 
of the respective IRS, and three elements describing possible unavailable transmission paths(because of outages) for use in the restoration plan[2].

The final element of each IRS is input of auto-encoder. The auto-encoder is just a explanation part of Monitoring and Identification and intelligent sensing and monitoring are the part of Computational Intelligence. Monitoring and control of any devices or subsystems in a smart grid depends on the quality and availability of sensor measurements. Corrupted or interrupted measurements, due to unexpected system conditions or malfunction of sensors, may result in incorrect operations of devices, and significantly affect the reliability of power systems. For if Protection devices like Protective relays, measurement integrity is essential to ensure the performances of these high-speed devices. Measurements devices like we use the instrument transformer in the power system. Instrument transformers are sometimes distorted due to saturation and transients during grid faults or changes in system operating points. ANN-based compensators were proposed to correct these measurement distortions and reconstruct the secondary measurements [6].The ANN was trained offline to learn the inverse function of a current transformer for different primary current conditions. In severe cases when sensor measurements are lost, a missing sensor restoration (MSR) scheme, using auto-associative neural networks(auto-encoder) and particle swarm optimizations(PSO), was proposed by Qiao[7]. So some important features are play the vital role in auto-encoder. The auto-encoder was trained to extract important features, and automatically establish the auto-correlations and crosscorrelations of sensor measurements. So After training, when one or more sensor measurements were lost, the remaining healthy data, together with the PSO estimated missind data, were fed into the auto-encoder. The error signal $\mathrm{E}_{\mathrm{S}}$ shown in fig. 1 was used as the fitness function for PSO. Once $E_{S}$ dropped to an acceptable level, the missing data were restored by $\mathrm{S}_{\mathrm{R}}$. So This MSR scheme was then further used by Qiao to develop a sensor-fault-tolerant controller for a static synchronous series compensator (SSSC) [7].The controllers are used in case of sensor failure to perform well even with multiple failures. The adaptive critic designs(ACDS) proposed by Werbos[3]are among the most advanced ones. ACDS combine the concept of reinforcement learning and approximate dynamic programming(ADP), and enable controllers to deal with nonlinear no stationary systems in the presences of noise and uncertainty[4],[5]. Nevertheless, the power grids have become so complex that the traditional way of control and operation, with poor visibility and significant human involvement, is facing major challenges. Issues of large-scale blackouts and energy security have driven the electric power industry to rethink the questions of what are the missions of the future power grids and what are the options to achieve them. Besides building more lines, are there any other ways to improve the power grids? A concept of "smart grids", aiming at improving power systems, reliability, security, sustainability, efficiency, flexibility and affordability, emerges to answer these questions. Smart grid research and development activities have been carried out worldwide. In the U.S., a federal Smart Grid Task Force was established to coordinate for Applications (renewable generation, distributed generation, Large-scale storage, plug-in hybrid electric vehicles, smart appliances, etc.) . Automated asset monitoring is the first step to understand the real-time conditions of a power system. For example, a power line thermal monitoring scheme will allow the system operator to know the exact safety margin of a power line, and to temporarily overload some power lines to ride through emergencies without exceeding their thermal limits. An echo-state network(ESN) approach was proposed by Yang to identify the power-line thermal dynamics and estimates the real-time line temperature directly from the current measurements[8]. diverse activities in the U.S. government related to smart grid technologies, practices and services. The European power networks, and to foster and support the development of smart grids in Europe. IEEE launched a smart grid website as an integrated gateway to smart grid standards, education, publications and news from IEEE. Power Quality monitoring and classification is another example of improving the visibility of power systems. While time-domain current and voltage measurements are often easy to obtain, CI techniques can help translate these measurements into useful power quality information. Two-stage power quality monitoring systems; which employed the wavelet transform for feature extraction and ANNs for power quality classification, have been reported [9]. A novel harmonic identifier using ESNs was proposed by Mazumdar to differentiate current harmonic contributions between a non linear load and a distorted voltage source[10].An ESN was first trained to identify the non linear load dynamics under the distorted voltage source, and then a pure sinusoidal signal was fed to the trained ESN to obtain the true current harmonic contribution of the non linear load. The proposed restoration scheme will present a restoration plan to the EMS operator following the occurrence of a wide area disturbance[11]. The power system operator must apply the all-open switch strategy through the SCADA/EMS or through regional control centers before the plan is implemented. The restoration plan provided by the proposed scheme will be composed of energizing sequences and restoration load percentage pick up values for all islands. As the final step of the total restoration, the closing of the tie-lines will be the responsibility of the system operator. The tie-lines should be closed when all the islands are restored and are in steady state. 


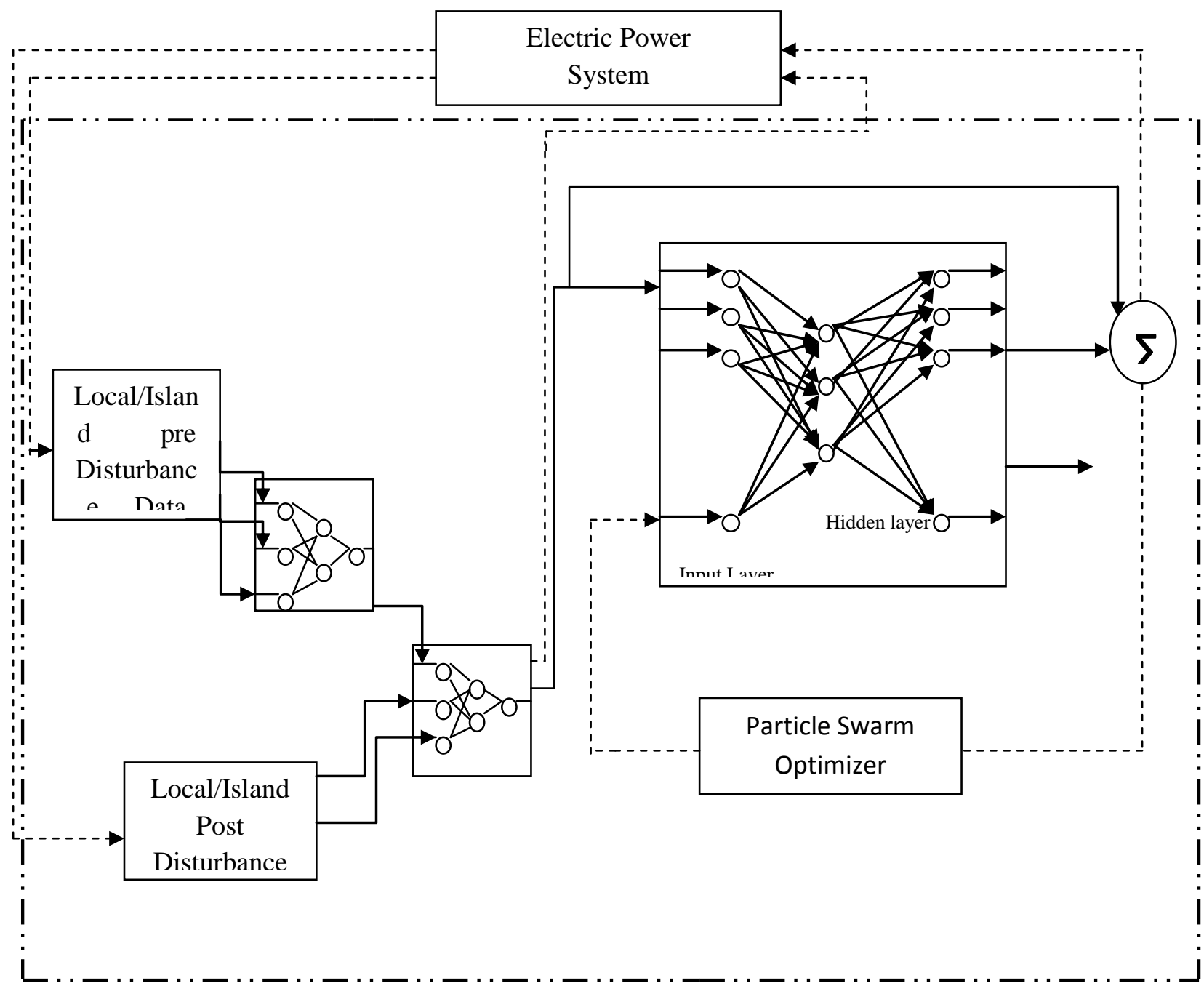

Fig. 1: Island restoration scheme(IRS) with Online restoration of missing sensor data $\left(S_{H}\right.$ denotes healthy data, $S_{M}$ denotes estimated data by PSO, and $S_{R}$ denotes restored sensor data)

\section{ALARM ANALYSIS FOR FAULT DIAGNOSIS}

Implementing the ES in EPS for the first time is encountered in the 1990s [12]. There are several fields where ES has been implemented: alarm analysis, fault diagnosis, load forecast, planning within the EPS, economic dispatch and production. The most widespread usage of ES is in analysing the alarm and diagnosing the failure, taking up $40 \%$ of its usage, while the planning is used in $15 \%$ of the cases [13]. ES method is shown in fig. 2: an incoming alarm, coming from rule-andfact knowledge base, forms the hypothesis and checks if the alarm matches particular lists for certain hypothesis. If it does, then it increases the hypothesis for one, and if it does not, then new hypothesis are formed including their related alarm lists. After being increased by one, the hypothesis is checked containing all the alarm signals. If the hypothesis has all the alarms, it gets printed out, if not, then it waits for the following alarm to do furthur assessment. It should be noted that this is a genetic algorithm(GA) which has all the genetic features: random data entry, a certain type of selection and iteration leading to the possible solution. Diagnostic methods usually use backward reasoning. Relay function can also be viewed as forward reasoning, one thing causing the other. Forward reasoning starts from the known facts towards the goal. Alarm's processor uses ES as the program to analyse appearing alarms during the fault in EPS. The alarm processor helps the operator to make decisions about the currently unknown black out areas after a breakdown of a certain part of EPS. The ES [14] method used in this paper, tries to organize huge amounts of disorganized data in the logical manner, extracting some diagnosis conclusions. It is important to emphasize that these are not standard numeric variables, but linguistic variables, making logical relationships for these linguistic variables in a standard procedural language such as $\mathrm{C}++$ requires enormous programming knowledge. Declarative programming is used for these kinds of problems. Alarm analysis is designed by using the expert system method making logically organized structure of failure diagnosis from basics data. A dynamic input data are given in the form of linguistic variables, therefore the solution is also a dynamic cluster of linguistic variables (hypothesis). The solution is changed in accordance with the incoming data, Incoming data stochastically arrive according to a generic algorithm which places hypotheses and comparisons in a certain criterion. A number of iterations in the generic algorithm are finite and determined by the number of input alarms. Knowledge base consists of network topology and rules for fault diagnosis. Network topology is presented by the facts and it is easily formed as network graph, whose nodes are easily interconnected. Rules for the fault location and fault diagnosis are obtained through interviews with people involved in relay protection. The rules are called cause-effect and can be 
defined for the components of the EPS to the parts of EPS to the parts of EPS.

There are many types of ES and they are different from one another in a way they organize knowledge in their knowledge base. These are three types: Rule Base, Case Base and Model Base. ES can be off-line and on-line. The ES based methods uses hypothesis method- the same method as humans applied when analysing alarms, just in a modified, automatic version. The hypothesis method begins from the final point (hypothesis) trying to include as many facts as possible. So that the hypothesis would stay active. The ES method is based on rules and principles which make hypothesis withholding predesigned fact clusters. The ES method is based on an algorithm which compares the rules.

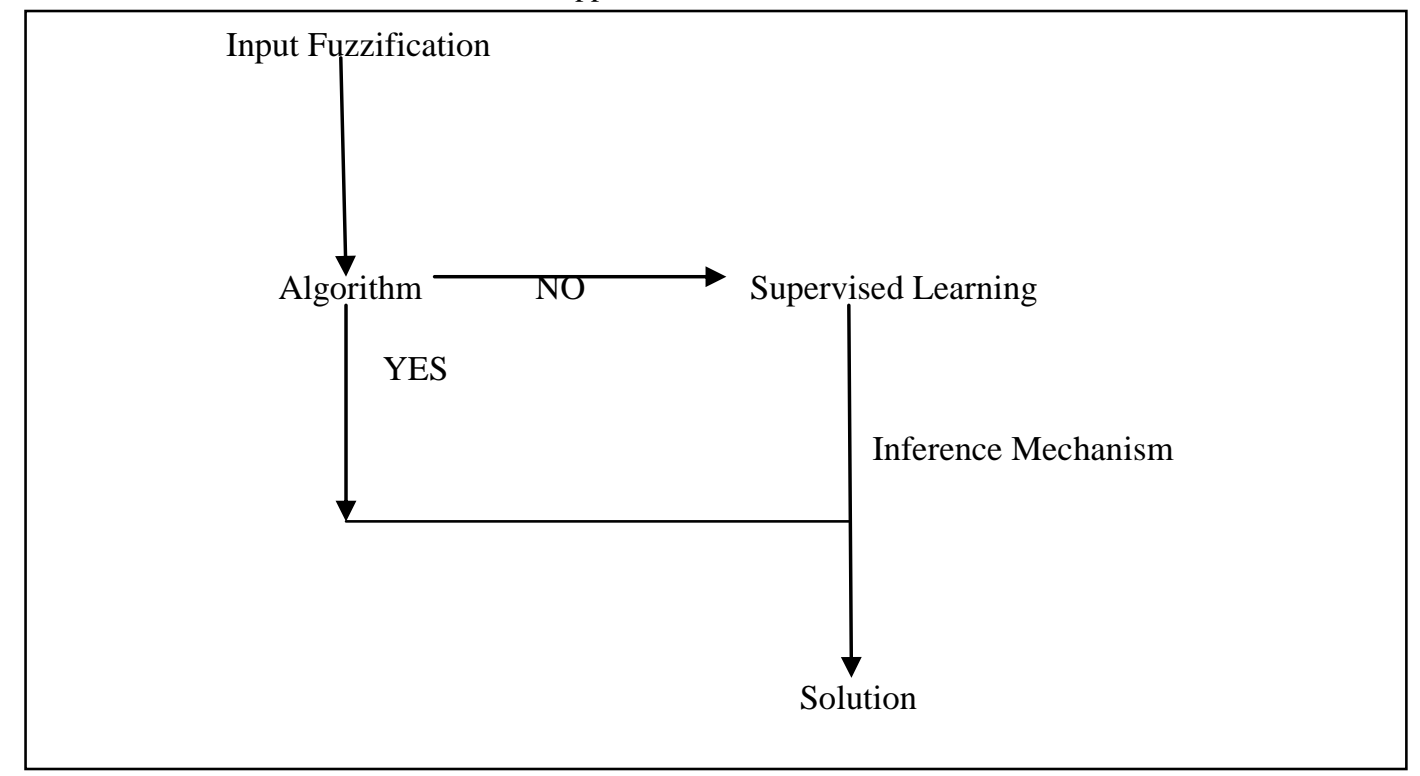

Fig. 2: Basic Diagram for Alarm Analysis for Fault Diagnosis

On the basis of fuzzy logic theory representation the alarm analysis in electrical power system based on expert system. First of all we have input fuzzification parameter and then it go for learning process. After the learning it go for alarm analysis. So by using the alarm analysis we do fault diagnosis. This Fault Diagnosis is used for load forecasting. So this is the theoretical explanation for alarm analysis for fault diagnosis in electrical power system based on expert system.

For Mathematical Expression, Suppose we have membership function which contain the different variables. For Example: we have

$$
\begin{aligned}
& \mathbf{P}_{\mathrm{Ti}}\left(\mathbf{F}_{\mathrm{i}}\right)=\left(\mathbf{X}_{\mathrm{i}}, \mathbf{Y}_{\mathrm{i}}\right)\left(\mathbf{X}_{\mathrm{j}}, \mathbf{Y}_{\mathrm{j}}\right)\left(\mathbf{X}_{\mathrm{k}}, \mathbf{Y}_{\mathrm{k}}\right) \\
& \mathbf{P}_{\mathrm{T} 1}\left(\mathbf{F}_{1}\right)=\left(\mathbf{X}_{1}, \mathbf{Y}_{1}\right)\left(\mathbf{X}_{2}, \mathbf{Y}_{2}\right)\left(\mathbf{X}_{3}, \mathbf{Y}_{3}\right) \\
& \mathbf{P}_{\mathrm{T} 2}\left(\mathbf{F}_{2}\right)=\left(\mathbf{X}_{4}, \mathbf{Y}_{4}\right)\left(\mathbf{X}_{5}, \mathbf{Y}_{5}\right)\left(\mathbf{X}_{6}, \mathbf{Y}_{6}\right)
\end{aligned}
$$

So We get the value of $\mathbf{P}_{\mathrm{T} 3}\left(\mathbf{F}_{3}\right)$

$$
\begin{aligned}
& P_{T 3}\left(F_{3}\right)=\left(X_{1}, Y_{1}\right)\left(X_{2}, Y_{2}\right)\left(X_{3}, Y_{3}\right) \\
& +\left(X_{4}, Y_{4}\right)\left(X_{5}, Y_{5}\right)\left(X_{6}, Y_{6}\right)
\end{aligned}
$$$$
2
$$

The following condition regulates the fact;

$$
\left|\theta_{\mathrm{A}^{--}} \boldsymbol{\theta}_{\mathrm{R}}\right|=\boldsymbol{\beta}
$$

First Condition is $\boldsymbol{\beta} \approx \mathbf{0}$

\section{Second Condition is $\boldsymbol{\beta} \gg \mathbf{0}$}

The second condition is only for alternate hypothesis. So this is a mathematical expression on the basis of fuzzy logic theory representation the alarm analysis in electrical power system based on expert system. We can also explain this in another way- Let $\mathrm{x} \sim$ unif $[0,2]$, What is the expected area of a square with side lenght $\mathrm{X}$. We consider the expected value of a linear transformation $\boldsymbol{\alpha} \mathbf{X}+\boldsymbol{\beta}$. The expected area is not equal to the square of the expected sidelenght. For the sidelenght small values(near 0 ) and large values(near 2) average each other out around 1. However, When the values are squared values near 0 but those near 2 end up closer to 4 . Thus, large values have more impact when squared and push the expected area up.

$$
\begin{aligned}
& \text { So } \quad \boldsymbol{\beta}=\mathbf{X}^{2} \\
& \begin{aligned}
\mathbf{P}_{\mathrm{T} 3}\left(\mathbf{F}_{3}\right)=\mathbf{P}(\boldsymbol{\beta} \leq \boldsymbol{\alpha}) \\
=\mathbf{P}(\mathbf{X} \leq \sqrt{ } \boldsymbol{\alpha}) ; 0 \leq \boldsymbol{\alpha} \leq 4
\end{aligned}
\end{aligned}
$$

\section{CONCLUSIONS}

The results significantly recommend the use of the computer as an operator aid instead of the use of predefined operating procedures for restoration. The restoration scheme with autoencoder requires multidisciplinary research and engineering efforts, and more importantly, intelligence and innovations in context to the aspects of electrical power engineering.

Alarm analysis could apply Expert systems with uncertainty have been applied in perspective to alarm analysis Based on 
all available data from SCADA, alarm analysis using ES is extremely efficient to handle the failure events in EPS thereby improving the decision making feature.

\section{REFERENCES}

[1] M. M. Adibi, "Power system restoration methodologies and implementation strategies," IEEE Press Power Eng. Series, 2000.

[2] M. M. Adibi, "Special considerations in power system restoration," IEEE trans. Power Syst., vol. 7 pp. 14191427, Nov. 1992.

[3] P. Werbos, "New directions in ACDs: keys to intelligent control and understanding the brain," in Proc. 2000 Int'l joint Conf. Neural Networks(IJCNN), vol. 3,pp,6166,Como,Italy,24 Jul,2000.

[4] D. Prokhorov and D.Wunsch, "adaptive critic designs," IEEE Trans. Neural Netw., vol.8,no.5,pp. 997-1007,Sep. 1997.

[5] J.Si, A. Barto, W.powell, and D. Wunsch, Hanbook of learning and approximate dynamic programming, WileyIEEE Press,2004.ISBN-13:978-0471660545.

[6] H. Khorashadi-Zadeh and Z. Li, "Intelligence Based Data Acquisition System for Protective Relays," in Proc. 2008 IEEE PES Transmission and Distribution Conf. And Exp., Chicago,IL,USA,Apr.2008.

[7] W. Qiao,G. Venayagamoorthy, R.Harley, " Missingsensor-fault-tolerant control for SSSC FACTS device with real-time implementation",IEEE Trans. Power Del.,vol.24,no.2,pp.740-750,apr.2009.

[8] Y. Yang, R. G. Harley,D. Divan, and T. G. Habetler, "Overhead Conductor Thermal dynamics Identification by using Echo State Networks," in Proc.2009 Int'1 Joint Conf. Neural Networks(IJCNN), Atlanta, Georgia, USA, Jun.14-19,2009.

[9] H.He and J. A. Starzyk, “ A Self Organizing Learning Array System for Power Quality Classification based on Wavelet Transform," IEEE Trans.Power Del.,vol.21,pp.286-295,January ,2006.

[10] J. Mazumdar and R. G. Harley, "Utilization of Echo State Networks for Differentiating Source and Non-linear load harmonics in the utility networks," IEEE Trans. Power Electron.,vol.23,no. 6,pp. 2738-2745,Nov.2008.

[11] "New approaches in power system restoration," IEEE Trans. Power Syst., vol.7,pp. 1428-1434,Feb.1992.

[12] Kezunovic, M: Luo,X. An Expert System for Diagnosis of Digital Relay Operation,Project T-17.

[13] Mcdonald, J. R. McArthur, S. Burt, G .M. Zielinski, J.Intelligent Knowledge Based system in Electrical poer Engineering, Chapman \& Hall, London,1997.

[14] Giarratano, J. Riley.G, Expert Systems Principles and programming, PWS Publishing Company,USA,1998. 